\title{
Computational modeling of rate-dependent domain switching in piezoelectric materials
}

\author{
A. Arockiarajan ${ }^{\text {a }}$, A. Menzel ${ }^{\mathrm{a}, *}$, B. Delibas ${ }^{\mathrm{a}}$,W. Seemann ${ }^{\mathrm{b}}$ \\ a Chair of Applied Mechanics, Department of Mechanical and Process Engineering, University of Kaiserslautern, \\ P.O. Box 3049, D-67653 Kaiserslautern, Germany \\ $\mathrm{b}$ Institute of Engineering Mechanics, Department of Mechanical Engineering, University of Karlsruhe, \\ Kaiserstr. 12, D-76131 Karlsruhe, Germany
}

Received 20 October 2005; accepted 23 January 2006

Available online 9 March 2006

\begin{abstract}
In this contribution a micromechanically motivated model for rate-dependent switching effects in piezoelectric materials is developed. The proposed framework is embedded into a three-dimensional finite element setting whereby each element is assumed to represent an individual grain. Related dipole (polarization) directions are thereby initially randomly oriented at the element level to realistically capture the originally un-poled state of grains in the bulk ceramics. The onset of domain switching processes is based on a representative energy criterion and combined with a linear kinetics theory accounting for time-dependent propagation of domain walls during switching processes. In addition, grain boundary effects are incorporated by making use of a macromechanically motivated probabilistic approach. Standard volume-averaging techniques with respect to the response on individual grains in the bulk ceramics are later on applied to obtain representative hysteresis and butterfly curves under macroscopically uniaxial loading conditions at different loading frequencies. It turns out that the simulations based on the developed finite element formulation nicely match experimental data reported in the literature.
\end{abstract}

(c) 2006 Elsevier Masson SAS. All rights reserved.

Keywords: Piezoelectricity; Rate-dependency; Domain switching; Coupled problems; Finite element method

\section{Introduction}

Piezoelectric materials exhibit coupled mechanical and electrical response which makes them widely used for the design of smart structures and intelligent systems as sensors and actuators. Common applications are, for instance, in the field of vibration damping and noise control, fuel injection mechanism in automobiles, ink-jet printers, micro-positioning, etc. These materials typically exhibit pronounced nonlinear response under sufficiently higher electromechanical loading conditions. The main reason for this type of behavior stems from the change in spontaneous polarization directions which is referred to as domain switching. This distortion in the lattice structure apparently causes a change in strains, so-called spontaneous strains. Moreover, domain walls which separate different phases

\footnotetext{
* Corresponding author.

E-mail address: amenzel@ rhrk.uni-kl.de (A. Menzel).

$U R L:$ http://mechanik.mv.uni-kl.de (A. Menzel).
} 
within individual grains, as typically observed in poly-crystalline piezoelectric materials, also move through the crystal so that the domain switching process is inherently inhomogeneous. Various types of experiments have been studied and reported in the literature in order to better understand the nonlinear behavior of these materials; the reader is referred to the contributions by, e.g., Cao and Evans (1993), Hwang et al. (1995), Lynch (1996), Lu et al. (1999) or Huber and Fleck (2004) and references cited therein, where the specimen of interest is usually subjected to quasi-static cyclic electric and/or mechanical loading.

Computational modeling of nonlinear response of piezoelectric materials can be classified into two major approaches: On the one hand, micromechanical formulations directly address fundamental physical insight of the material. When setting up such theories, different length scales according to which particular aspects of the material behavior and substructure can be incorporated into the model; compare, for instance, Hwang et al. (1995), Chen and Lynch (1998) or Lu et al. (1999). In general, these models account for the bulk piezoelectric material being composed of various randomly oriented sub-cells. Commonly, volume averaging techniques are then applied to analyze the macroscopic behavior of representative specimens. Concerning the switching process itself, initialization of domain switching is, for instance, initiated if the reduction of potential energy exceeds a certain threshold; compare Hwang and McMeeking (1998a, 1998b). Alternative formulations, e.g., based on volume fraction concepts are elaborated by Chen et al. (1997) among others. Li and Weng (2001) recently proposed a formulation wherein thermodynamic considerations motivated the reduction in Gibbs free energy to serve as the driving force for switching. A critical switching value of the electric displacement for mono-crystalline piezoceramics together with additional stability arguments has been elaborated by Fotinich and Carman (2000). On the other hand, phenomenological or rather macromechanical approaches constitute an alternative modeling concept. A representative set of state variables is thereby adopted and incorporated into essential expressions and functions such as the free energy. Further constitutive relations and evolution equations are then established by taking fundamental thermodynamic considerations into account; see for instance Lynch and McMeeking (1994), Kamlah and Tsakmakis (1999), Landis (2002) or Schröder and Romanowski (2005). When setting up such phenomenologically motivated theories, one generally has the choice whether to fix material properties or to allow the material properties to change during the deformation process.

In general, the behavior of piezoelectric materials turns out to be rate-dependent which means that domains at the micro-level require a certain time to switch. This property obviously also affects the overall macroscopic response of piezoelectric materials. Janta (1971) developed a model to estimate the influence of the domain shape and different loading frequencies on the amplitude of the hysteresis loop as well as on the coercive electric field. The reported results indicate that the coercive electric field is solely slightly affected by the shape of the domain structure itself. In this context, Abeyaratne et al. (1994) developed a free energy based model and made use of a kinetic relation in order to set up a nucleation criterion. Switching response of individual phases are thereby captured by means of a constitutive theory. Furthermore, Arlt (1996a, 1996b) proposed a modeling framework within which the propagation of new phases is dominated by the phase boundaries between the old and new nucleated phases. Rate-dependent response at excitation frequency ranges including domain wall motion has been addressed by Smith and Ounaies (2000).

The finite element method turned out as a powerful tool for numerical simulations of piezoelectric materials. Fundamentals for the problem at hand have been addressed by Allik and Hughes (1970), wherein use of variational principles has been made. Subsequently, (nonlinear) finite element formulations have been investigated in different contexts; see for instance the coupled formulations in Gaudenzi and Bathe (1995) and Schröder and Gross (2004) or, for an overview, the monograph by Silvester and Ferrari (1996). In general, finite element techniques can be used to simulate both micro- and macromechanically motivated models, see for instance Hwang and McMeeking (1998a, 1998b) or Kamlah and Böhle (2001). However, applying the finite element method to micromechanical models with very fine meshes so that representative substructures are captured within a realistic macroscopic specimen size, might need too much CPU time compared with macromechanical models. Consequently, the development of powerful (coupled) homogenization techniques seems to be of particular interest.

The main goal of the present study is the development of a three-dimensional coupled finite element model, extending previous elaborations by the authors reported in Arockiarajan et al. (2005a, 2005b), to electromechanically induced rate-dependent domain switching of piezoelectric materials. The onset of domain switching is thereby initiated by means of an energy-based criterion. Moreover, nucleation of grains and propagation of domain walls are captured via a linear kinetics theory. Intergranular grain boundary effects are phenomenologically modeled by combining the switching criterion with an additional switching probability function which, practically speaking, weights a 
critical energy barrier. Finite element simulations as based on the proposed model including standard volume averaging techniques are compared with experimentally measured hysteresis and butterfly curves reported in the literature.

The paper is organized as follows: Section 2 briefly reviews essential balance relations and constitutive equations for piezoelectric materials. The modeling of rate-independent and in particular rate-dependent domain switching as well as the incorporation of intergranular effects are addressed in Section 3. Numerical examples as based on a coupled finite element formulation, which is summarized in Appendix A, are presented in Section 4. Finally, a short summary concludes the paper, see Section 5.

\section{Constitutive model}

For operating temperatures above the Curie temperature the symmetry of a piezoelectric material might change, depending on the particular composition of the material, from simple cubic structures to tetragonal or rhombohedral symmetries. Within this ferroelectric phase, relative atom positions in the crystal lattice might change which gives rise to nonzero net dipole moments. The effect itself is commonly denoted as piezoelectric effect. Correlated net dipole moments (per unit volume) are usually introduced as spontaneous polarization (vectors). During such phase transitions, the movements of atoms apparently result in lattice distortions which consequently cause mechanical strains, so-called spontaneous strains. In the following, we briefly summarize essential balance relations and constitutive equations for piezoelectric materials with particular emphasis on the physical phenomena mentioned above and appropriately capturing the initially un-poled state.

\subsection{Balance relations}

Assume $B$ to denote the continuum body of interest. Moreover, $\mathcal{B} \subset \mathbb{R}^{3}$ denotes the configuration of $B$ as defined by placements $\boldsymbol{x} \in \mathbb{R}^{3}$ of material points. The electric potential $\phi \in \mathbb{R}$ and the displacement field $\boldsymbol{u} \in \mathbb{R}^{3}$ represent the underlying essential degrees of freedom. Equilibrium of $B$ and the corresponding Gauß equation consequently result in

$$
\begin{array}{llll}
\mathbf{0}=\nabla \cdot \boldsymbol{\sigma}+\boldsymbol{b} & \text { in } \mathcal{B}, & 0=\nabla \cdot \boldsymbol{D}-\rho_{f} & \text { in } \mathcal{B}, \\
\boldsymbol{u}=\boldsymbol{u}^{\mathrm{p}} & \text { on } \partial \mathcal{B}_{u}, & \phi=\phi^{\mathrm{p}} & \text { on } \partial \mathcal{B}_{\phi}, \\
\boldsymbol{t}=\boldsymbol{t}^{\mathrm{p}}=\boldsymbol{\sigma} \cdot \boldsymbol{n}_{\sigma} & \text { on } \partial \mathcal{B}_{\sigma}, & -q=-q^{\mathrm{p}}=\boldsymbol{D} \cdot \boldsymbol{n}_{D} & \text { on } \partial \mathcal{B}_{D}
\end{array}
$$

with $\partial \mathcal{B}_{u} \cup \partial \mathcal{B}_{\sigma}=\partial \mathcal{B}_{\phi} \cup \partial \mathcal{B}_{D}=\partial \mathcal{B}$ and $\partial \mathcal{B}_{u} \cap \partial \mathcal{B}_{\sigma}=\partial \mathcal{B}_{\phi} \cap \partial \mathcal{B}_{D}=\emptyset$; see Fig. 1 for a graphical illustration. The electric displacement vector and the stress tensor have thereby been denoted as $D \in \mathbb{R}^{3}$ and $\sigma=\sigma^{\mathrm{t}} \in \mathbb{R}^{3 \times 3}$. Corresponding unit normals with respect to the underlying surfaces of interest are characterized by $\boldsymbol{n}_{\sigma, D} \in \mathbb{R}^{3}$.

\subsection{Constitutive equations}

The commonly adopted set of so-called nonlinear constitutive equations for piezoelectric materials, accounting for spontaneous polarization $\boldsymbol{P}^{\mathrm{s}} \in \mathbb{R}^{3}$ and trace-less spontaneous strains $\boldsymbol{\varepsilon}^{\mathrm{s}}=\left[\boldsymbol{\varepsilon}^{\mathrm{s}}\right]^{\mathrm{t}} \in \mathbb{R}^{3 \times 3}$, reads
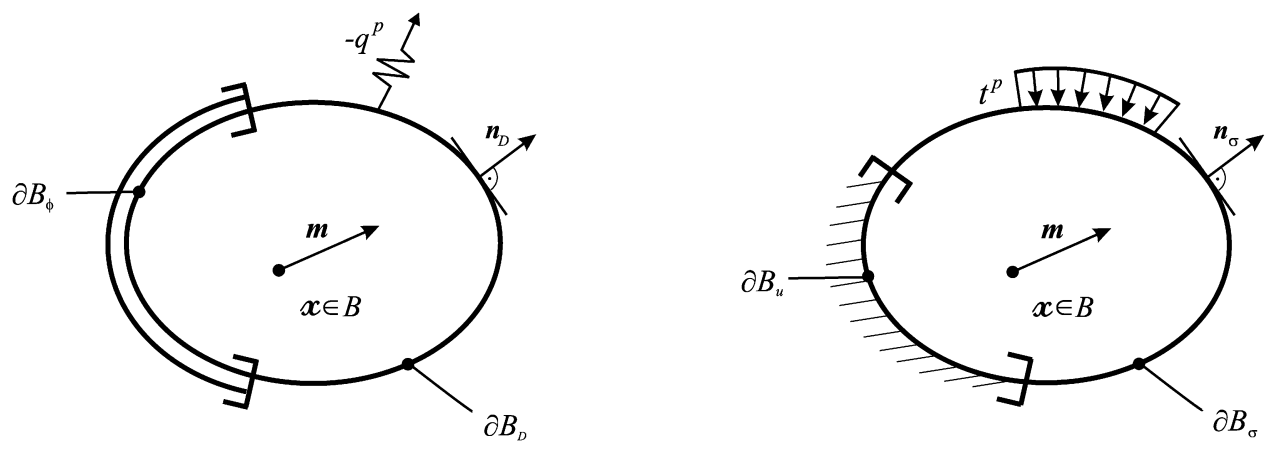

Fig. 1. Configuration and boundary conditions for the electrical and mechanical problem. 


$$
\sigma=\mathrm{C}:\left[\boldsymbol{\varepsilon}-\boldsymbol{\varepsilon}^{\mathrm{s}}\right]-\boldsymbol{E} \cdot \boldsymbol{d}, \quad \boldsymbol{D}=\boldsymbol{d}:\left[\boldsymbol{\varepsilon}-\boldsymbol{\varepsilon}^{\mathrm{s}}\right]+\epsilon \cdot \boldsymbol{E}+\boldsymbol{P}^{\mathrm{s}}
$$

wherein $\boldsymbol{E}=-\nabla \phi \in \mathbb{R}^{3}$ represents the electric field and $\boldsymbol{\varepsilon}=\boldsymbol{\varepsilon}^{\mathrm{t}}=\nabla^{\mathrm{sym}} \boldsymbol{u} \in \mathbb{R}^{3 \times 3}$ denotes the strain tensor. Material constants are introduced by means of the elastic stiffness $\mathbf{C}=\mathbf{C}^{t}=\mathbf{C}^{\mathrm{T}} \in \mathbb{R}^{3 \times 3 \times 3 \times 3}$, the dielectric permittivity $\boldsymbol{\epsilon}=$ $\boldsymbol{\epsilon}^{\mathrm{t}} \in \mathbb{R}^{3 \times 3}$ and the piezoelectric tensor $\boldsymbol{d} \in \mathbb{R}^{3 \times 3 \times 3}$, respectively. From the physical point of view, material properties, and in particular anisotropic characteristics stemming from deformation-induced reorientation of the underlying unitcells, change during the entire deformation process. In the following, we, nevertheless, adopt an isotropic model for the elastic stiffness and dielectric permittivity, namely

$$
\mathbf{C}=\lambda \boldsymbol{I} \otimes \boldsymbol{I}+2 \mu \mathbf{l}^{\mathrm{sym}}, \quad \boldsymbol{\epsilon}=\epsilon \boldsymbol{I}
$$

with $\lambda$ and $\mu$ denoting the Lamé parameters, $\epsilon$ basically defining the dielectric permittivity and $\boldsymbol{I}$ as well as $\mathbf{I}$ abbreviating second and fourth order identity tensors. Directional dependencies of the piezoelectric tensor, however, are addressed by directly including the polarization orientation so that $\boldsymbol{d}$ accounts for transversely isotropic response of perovskite crystallites with tetragonal microstructure; to be specific

$$
\boldsymbol{d}=d_{33} \boldsymbol{M}+d_{31}[\boldsymbol{m} \otimes \boldsymbol{I}-\boldsymbol{M}]+d_{15}\left[\frac{1}{2}[\boldsymbol{I} \otimes \boldsymbol{m}+\boldsymbol{I} \bar{\otimes} \boldsymbol{m}]-\boldsymbol{M}\right]
$$

wherein $\boldsymbol{m} \in \mathbb{R}^{3},\|\boldsymbol{m}\|=1, \boldsymbol{M}=\boldsymbol{m} \otimes \boldsymbol{m} \otimes \boldsymbol{m}$ and $[\boldsymbol{I} \otimes \boldsymbol{m}+\boldsymbol{I} \bar{\otimes} \boldsymbol{m}]: \boldsymbol{a}=[\boldsymbol{I} \otimes \boldsymbol{m}+\boldsymbol{I} \bar{\otimes} \boldsymbol{m}]: \boldsymbol{a}^{\mathrm{t}} \forall \boldsymbol{a} \in \mathbb{R}^{3 \times 3}$, respectively. Apparently, the piezoelectric tensor might vary from domain to domain with the unit-cell orientations $\boldsymbol{m}$ being aligned according to the polarization vector, i.e. $\boldsymbol{m}=\boldsymbol{P}^{\mathrm{s}} /\left\|\boldsymbol{P}^{\mathrm{s}}\right\|$. For the symmetry class $4 m m$ of interest in this work, $\boldsymbol{m}$ is in the $\langle 100\rangle$ family so that six different orientations (at fixed principal directions of the unit-cell) come into the picture.

\subsection{Random polarization initialization}

The virgin or rather un-poled state of piezoelectric materials is characterized by randomly oriented or in fact equally distributed polarization vectors. Consequently, the initial macroscopic response possesses almost isotropic properties since the initial polarization of the bulk piezoceramics vanishes at the macroscopic level. In view of the poly-crystalline substructure, individual grains are subdivided into several domains equipped with possibly different polarization directions. Under sufficiently high loading conditions, however, the polarization directions might reorient, see Fig. 2 for a graphical illustration. The deformation-induced polarization reorientation is addressed in the subsequent section, while we now focus on how the initial un-poled state is taken into account within the proposed finite element framework.

For the computation of the initial unit-cell orientation $\boldsymbol{m}$, which also determines the initial polarization vector $\boldsymbol{P}^{\mathrm{s}}$ as well as the initial spontaneous strains $\boldsymbol{\varepsilon}^{\mathrm{s}}$, commonly applied Eulerian angles $(\Phi, \Theta, \Psi)$ are adopted; see for instance the monograph by Goldstein et al. (2002) for detailed background information. To be specific, $\Phi, \Psi \in[0,2 \pi)$ and $\sin \left(\Theta-\frac{\pi}{2}\right) \in[-1,1]$ are generated by random. The polarization direction consequently takes, e.g., the format

$$
\boldsymbol{m}=[\mathrm{s}(\Phi) \mathrm{c}(\Psi)+\mathrm{c}(\Theta) \mathrm{c}(\Phi) \mathrm{s}(\Psi)] \boldsymbol{e}_{1}-[\mathrm{s}(\Phi) \mathrm{s}(\Psi)-\mathrm{c}(\Theta) \mathrm{c}(\Phi) \mathrm{c}(\Psi)] \boldsymbol{e}_{2}-[\mathrm{s}(\Theta) \mathrm{c}(\Phi)] \boldsymbol{e}_{3}
$$

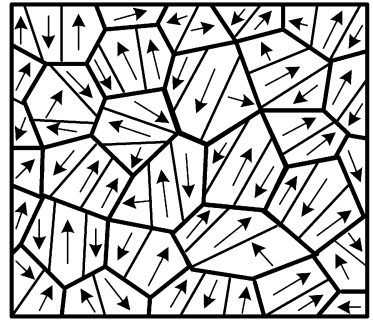

Before loading

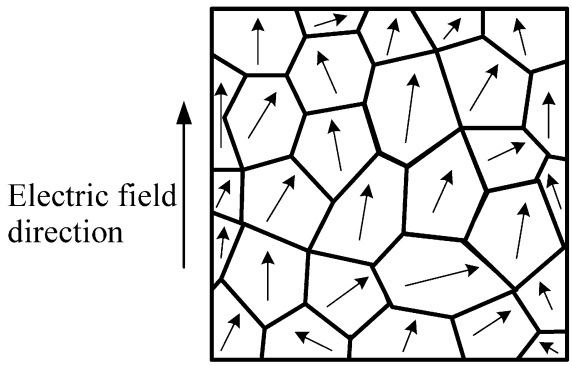

After loading

Fig. 2. Two-dimensional graphical illustration of the un-poled virgin state (left) and the fully-poled state (right) after electrical loading of a poly-crystalline piezoelectric material. 
wherein use of the abbreviations $\mathrm{c}(\bullet)$ and $\mathrm{s}(\bullet)$ for $\cos (\bullet)$ and $\sin (\bullet)$ has been made and, moreover, $\left\{\boldsymbol{e}_{1,2,3}\right\}$ denotes a space-attached Cartesian frame. The initialization of the spontaneous strain tensor $\boldsymbol{\varepsilon}^{\mathrm{s}}$ additionally requires providing an orthonormal frame including $\boldsymbol{m}$, say $\left\{\boldsymbol{m}_{1}, \boldsymbol{m}_{2}, \boldsymbol{m}_{3}=\boldsymbol{m}_{1} \times \boldsymbol{m}_{2}\right\}$. Choosing $\boldsymbol{m}_{2}=\boldsymbol{m}$, we observe that $\boldsymbol{m}_{1}$ allows, by analogy with Eq. (5), representation as

$$
\boldsymbol{m}_{1}=[\mathrm{c}(\Phi) \mathrm{c}(\Psi)-\mathrm{c}(\Theta) \mathrm{s}(\Phi) \mathrm{s}(\Psi)] \boldsymbol{e}_{1}-[\mathrm{c}(\Phi) \mathrm{s}(\Psi)+\mathrm{c}(\Theta) \mathrm{s}(\Phi) \mathrm{c}(\Psi)] \boldsymbol{e}_{2}+[\mathrm{s}(\Theta) \mathrm{s}(\Phi)] \boldsymbol{e}_{3}
$$

so that initial spontaneous strains can be introduced via

$$
\boldsymbol{\varepsilon}^{\mathrm{s}}=\varepsilon^{\mathrm{s}}\left[\boldsymbol{m}_{2} \otimes \boldsymbol{m}_{2}-\frac{1}{2}\left[\boldsymbol{m}_{1} \otimes \boldsymbol{m}_{1}+\boldsymbol{m}_{3} \otimes \boldsymbol{m}_{3}\right]\right] .
$$

Obviously, nonvanishing initial contributions of $\boldsymbol{P}^{\mathrm{s}} \neq \mathbf{0}$ and $\boldsymbol{\varepsilon}^{\mathrm{s}} \neq \mathbf{0}$ give rise to (inhomogeneous) electric displacements and initial mechanical stresses.

\section{Rate-dependent domain switching}

The polarization vector is generally assumed to be aligned with the orientation of the unit-cell. Since these directions are, for the tetragonally microstructured problem at hand, in the $\langle 100\rangle$ family, solely $90^{\circ}$ or $180^{\circ}$ domain switching occurs. Practically speaking, switching effects might reorient the actual polarization vector either according to $\left.\boldsymbol{m}_{n+1} \in\left\{ \pm \boldsymbol{m}_{1}, \pm \boldsymbol{m}_{3}\right\}\right|_{t_{n}}\left(90^{\circ}\right.$ switching) or subjected to $\left.\boldsymbol{m}_{n+1} \in\left\{-\boldsymbol{m}_{2}\right\}\right|_{t_{n}}\left(180^{\circ}\right.$ switching), respectively, whereby $\Delta t=t_{n+1}-t_{n}>0$ refers to a finite time interval.

\subsection{Energy-based switching criterion}

In the progression of this work, possible reduction in free energy $(\Delta U)$ is assumed to initiated domain switching processes. Conceptually speaking, switching processes come into the picture as soon as a particular (change in) energy barrier is exceeded, namely if

$$
\Delta U(\boldsymbol{u}, \phi)+\Delta \psi_{\mathrm{c}} \leqslant 0
$$

is violated. The ansatz is adopted (together with boundary conditions) from the contribution by McMeeking and Hwang (1997) wherein $\Delta \psi_{\mathrm{c}}$ denotes the particular (constant) energy barrier per unit volume. If switching occurs, the spontaneous polarization vector $\boldsymbol{P}^{\mathrm{s}}$ is assumed to adjust its direction $\boldsymbol{m}$ but to retain its magnitude $P^{\mathrm{s}}=\left\|\boldsymbol{P}^{\mathrm{s}}\right\|$. Accordingly, the polarization change $\Delta \boldsymbol{P}^{\mathrm{s}}$ satisfies

$$
\boldsymbol{P}_{n+1}^{\mathrm{s}}=\boldsymbol{P}_{n}^{\mathrm{s}}+\Delta \boldsymbol{P}^{\mathrm{s}} \quad \text { with } \Delta \boldsymbol{P}^{\mathrm{s}}=P^{\mathrm{s}}\left[\boldsymbol{m}_{n+1}-\boldsymbol{m}_{n}\right] .
$$

The jump in spontaneous strains is chosen so that its principal directions remain constant, i.e. generally persist aligned with the uni-cell orientation. Consequently, principal spontaneous strain values are allowed to shift. Incorporation of the polarization orientation as the predominant principal spontaneous strain direction consequently results in the representation

$$
\boldsymbol{\varepsilon}_{n+1}^{\mathrm{s}}=\boldsymbol{\varepsilon}_{n}^{\mathrm{s}}+\Delta \boldsymbol{\varepsilon}^{\mathrm{s}} \quad \text { with } \Delta \boldsymbol{\varepsilon}^{\mathrm{s}}=\frac{3}{2} \varepsilon^{\mathrm{s}}\left[\boldsymbol{m}_{n+1} \otimes \boldsymbol{m}_{n+1}-\boldsymbol{m}_{n} \otimes \boldsymbol{m}_{n}\right]
$$

wherein $\varepsilon^{\mathrm{s}}=\sqrt{2 / 3}\left\|\varepsilon^{\mathrm{s}}\right\|$ and $\operatorname{tr}\left(\boldsymbol{\varepsilon}^{\mathrm{s}}\right)=0$ being obvious. With these kinematic or rather compatibility considerations in hand, we next adopt the approach advocated by Hwang et al. (1995) for the energy-base switching criterion, namely

$$
\Delta U=\boldsymbol{E} \cdot \Delta \boldsymbol{P}^{\mathrm{s}}+\boldsymbol{\sigma}: \Delta \boldsymbol{\varepsilon}^{\mathrm{s}}>2 E_{0} P_{0} \quad \text { with } E_{0}, P_{0}>0 .
$$

The critical energy barrier $V_{\mathrm{c}} \Delta \psi_{\mathrm{c}}$ is thereby directly related to the coercive electric field $E_{0}$ and the critical polarization value $P_{0}$. Nevertheless, not only one but several switched polarization states might meet the criterion in Eq. (11) within one (specific) load step. The subsequently applied algorithm therefore incorporates the particular switched state corresponding to the largest amount in energy reduction which, however, might still violate Eq. (11). The applied criterion nicely serves for numerical simulations of rate-independent and rate-dependent switching embedded into a robust iterative finite element setting. 


\subsection{Rate-dependencies based on a volume fraction concept}

The domain switching framework elaborated above accounts for rate-independent phase transformations, i.e. until now it has been assumed that the switching process can be completed within the considered time interval $\Delta t$. From the physical point of view domain switching requires a finite interval of time which might be larger than the particularly chosen value for $\Delta t$; see Fig. 3 of a graphical illustration of domain wall motion. Moreover, these effects influence the overall macroscopic response so that rate-dependency is also observe at the macro-level. To give an example, cyclic loading experiments result in different hysteresis and butterfly curves for different loading frequencies.

In the progression of this work, a linear kinetics theory for the propagation of new phases through the material of interest is adopted; for a detail discussion the reader is referred to Delibas et al. (2006) and Arockiarajan et al. (2005a). In addition to the initiation of nucleation as based on Eq. (11) a critical time duration for the switching process is introduced. Practically speaking, if the time interval $\Delta t$, relating two subsequent incremental load steps, is larger than this critical time period, the phase boundary is enabled to completely propagate through the domain so that the switching process can be completed. Otherwise, the relation between $\Delta t$ and the critical time duration is used to determine the volume fraction which switches. To be specific, this limit time parameter $\Delta t_{1}$ is assumed to be defined by the ansatz

$$
\Delta t_{1}=\frac{C}{\|\boldsymbol{E}\|} \quad \text { with } C=\beta d>0
$$

being a temperature dependent parameter, compare Merz (1954). For purpose of illustration, we refer to a blocktype specimen under macroscopically uni-axial loading conditions in the following. The electric potential is thereby prescribed at the top and bottom surfaces, $\phi_{\text {top }} \neq 0$ but $\phi_{\text {bot }}=0$; compare Fig. 5. In this regard, let $f_{\phi}=1 / T_{\phi}$ denote the frequency of the applied electric potential which is linearly increased and decreased in time. Consequently, $T_{\phi}$
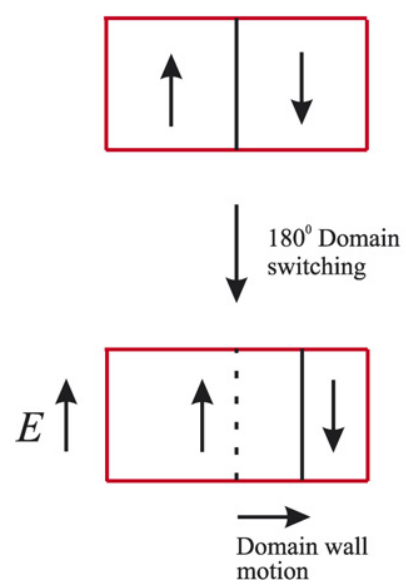

Fig. 3. Two-dimensional graphical illustration of domain wall motion.
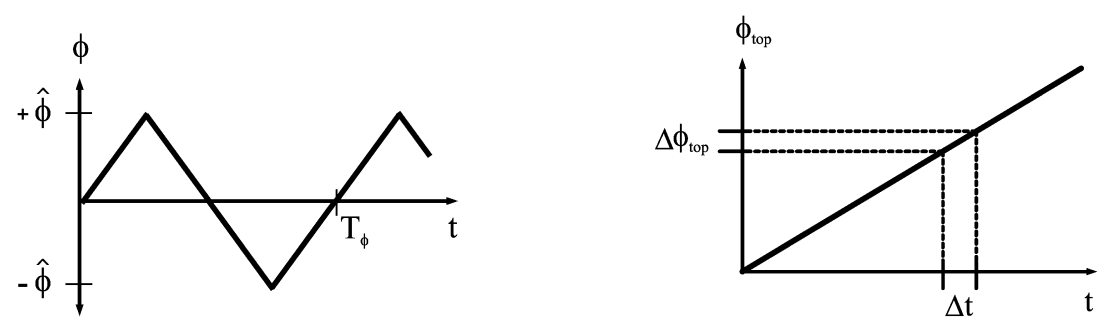

Fig. 4. Macroscopically uni-axial loading condition: triangular cyclic electrical loading (left) and representative incremental electrical loading step (right). 


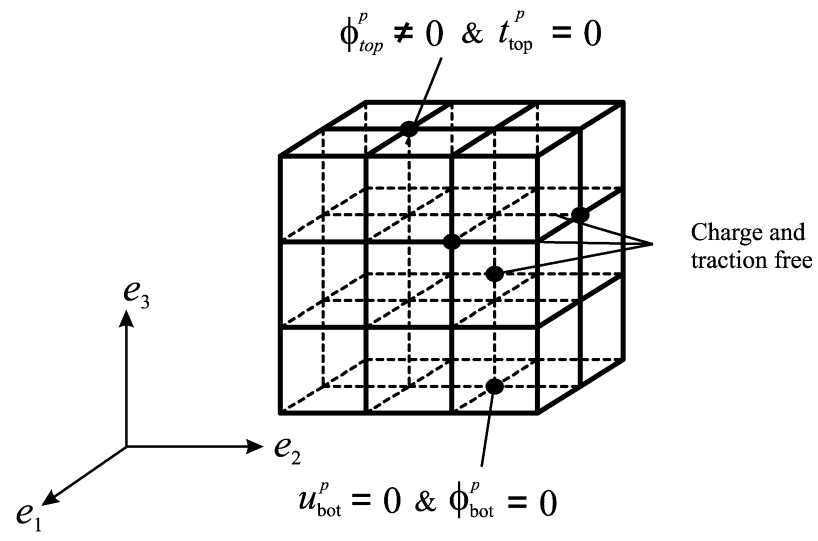

Fig. 5. Boundary conditions applied to the finite element discretization of the block-like specimen.

characterizes the time period of a full loading cycle, see Fig 4. Having these particular loading conditions in mind, the rate of the electric potential $\dot{\phi}_{\text {top }}$ allows representation as

$$
\dot{\phi}_{\text {top }}= \pm 2 \hat{\phi} /\left[\frac{1}{2} T_{\phi}\right]= \pm 4 \hat{\phi} / T_{\phi}= \pm 4 \hat{\phi} f_{\phi}
$$

wherein $\hat{\phi}>0$ characterizes the loading amplitude of the electric potential. Conceptually speaking, incremental loading steps of the electric potential $\Delta \phi_{\text {top }}$ are prescribed and consequently render essential boundary conditions; compare Fig. 4. Apparently, the correlated time interval takes the format

$$
\Delta t=\Delta \phi_{\text {top }} / \dot{\phi}_{\text {top }}=\Delta \phi_{\text {top }} /\left[4 \hat{\phi} f_{\phi}\right]
$$

from which we observe that $\Delta t$ decreases for increasing loading frequencies $f_{\phi}$. Within the subsequent numerical examples, $\Delta \phi_{\text {top }}$ and $\hat{\phi}$ are assumed to remain constant. If, however, $\Delta t<\Delta t_{1}$ and switching has been activated, the entire phase transformation process is (physically) not completed within $\Delta t$. Consequently, we accept that solely a fraction of the domain switches as represented by the ratio $\tau$. In practice, closing the phase transition might need several time steps $\Delta t$ if, for instance, rather large loading frequencies $f_{\phi}$ are applied. With this framework of a linear kinetics theory in hand, we next choose a particular ansatz for the volume fraction defining the switching subdomain $\Delta V$ with respect to the overall domain volume $V$. In this regard, let

$$
\Delta V=V \frac{\Delta t_{\mathrm{p}}}{\Delta t_{1}} \quad \text { which motivates } \quad \tau=\frac{\Delta V}{V}=\frac{\Delta t_{\mathrm{p}}}{\Delta t_{1}}
$$

wherein $\Delta t_{\mathrm{p}} \leqslant \Delta t_{1}$ denotes the time elapsed since the nucleation process has been initiated (for a particular load step). For $\Delta t_{1}>\Delta t$ we, accordingly, perform a linear interpolation between the totally switched $\left(\widehat{\boldsymbol{P}}_{n+1}^{\mathrm{s}}, \hat{\boldsymbol{\varepsilon}}_{n+1}^{\mathrm{s}}\right)$ and un-switched state $\left(\boldsymbol{P}_{n}^{\mathrm{s}}, \boldsymbol{\varepsilon}_{n}^{\mathrm{s}}\right)$ with respect to the volume fraction ratio $\tau$, namely

$$
\boldsymbol{P}_{n+1}^{\mathrm{s}}=\tau \widehat{\boldsymbol{P}}_{n+1}^{\mathrm{s}}+[1-\tau] \boldsymbol{P}_{n}^{\mathrm{s}}, \quad \boldsymbol{\varepsilon}_{n+1}^{\mathrm{s}}=\tau \hat{\boldsymbol{\varepsilon}}_{n+1}^{\mathrm{s}}+[1-\tau] \boldsymbol{\varepsilon}_{n}^{\mathrm{s}} .
$$

The computation of $\widehat{\boldsymbol{P}}_{n+1}^{\mathrm{s}}$ and $\hat{\boldsymbol{\varepsilon}}_{n+1}^{\mathrm{s}}$ is thereby directly based on Eqs. (9), (10). The rate-independent case, as developed in Eqs. (9), (10), is naturally included by setting $\tau=1$ for $\Delta t \geqslant \Delta t_{1}$.

Remark 3.1. The modified update scheme in Eq. (16) does, apparently, not preserve the norm of $\boldsymbol{P}^{\mathrm{s}}$ and $\boldsymbol{\varepsilon}^{\mathrm{s}}$. An alternative algorithmic implementation is provided by, e.g., $\boldsymbol{m}_{2}=\boldsymbol{P}^{\mathrm{s}} /\left\|\boldsymbol{P}^{\mathrm{s}}\right\|, \boldsymbol{P}^{\mathrm{s}} \leftrightarrow P^{\mathrm{s}} \boldsymbol{m}_{2}$ and $\boldsymbol{\varepsilon}^{\mathrm{s}}=\varepsilon^{\mathrm{s}}\left[\boldsymbol{m}_{2} \otimes \boldsymbol{m}_{2}-\right.$ $\left.\frac{1}{2}\left[\boldsymbol{m}_{1} \otimes \boldsymbol{m}_{1}+\boldsymbol{m}_{3} \otimes \boldsymbol{m}_{3}\right]\right]$, compare Eq. (7), which will numerically be investigated in future research.

\subsection{Grain boundary effects}

Individual grains in poly-crystalline piezoelectric materials are interconnected with each other at the grain boundaries. These boundaries particularly affect switching processes inside individual grains which is commonly denoted 
as so-called intergranular or grain boundary effects. To give an example, compatibility constraints might result in high stress levels nearby grain boundaries so that switching might be initiated under macroscopic loading levels at which phase transitions would otherwise be rather unexpected. Moreover, the initial random orientation of the polarization vectors further supports local electromechanical loading levels which can considerably vary from a macroscopic mean value. In summary, due to intergranular effects, domain switching usually occurs at loading levels well below the, e.g., macroscopic coercive electric field. Consequently, nonlinear response of piezoelectric materials is experimentally also within small electromechanical loading ranges observed. We will phenomenologically address this relevant modeling aspect by means of an additional switching probability function $P$, i.e

$$
P= \begin{cases}{\left[\frac{\overline{\boldsymbol{E}}^{\mathrm{e}} \cdot \Delta \boldsymbol{P}^{\mathrm{se}}+\overline{\boldsymbol{\sigma}}^{\mathrm{e}}: \Delta \boldsymbol{\varepsilon}^{\mathrm{se}}}{2 E_{0} P_{0}}\right]^{k}} & \text { for } \bar{E}^{\mathrm{e}}<E_{0}, \\ 1 & \text { for } \bar{E}^{\mathrm{e}} \geqslant E_{0}\end{cases}
$$

wherein $\bar{E}^{\mathrm{e}}=\left\|\overline{\boldsymbol{E}}^{\mathrm{e}}\right\|$ and the material parameter $k \geqslant 0$ allows calibration of simulations with experimental data. Moreover, use has thereby been made of volume-averaged quantities with respect to individual domains as represented by single finite elements within the subsequent numerical examples, namely

$$
\overline{\boldsymbol{E}}^{\mathrm{e}}=\frac{1}{\mathcal{V}^{\mathrm{e}}} \int_{\mathcal{V}^{\mathrm{e}}} \boldsymbol{E}^{\mathrm{e}} \mathrm{d} v, \quad \overline{\boldsymbol{\sigma}}^{\mathrm{e}}=\frac{1}{\mathcal{V}^{\mathrm{e}}} \int_{\mathcal{V}^{\mathrm{e}}} \boldsymbol{\sigma}^{\mathrm{e}} \mathrm{d} v
$$

so that the finally applied energy-based switching criterion, compare Eq. (11), results in

$$
\overline{\boldsymbol{E}}^{\mathrm{e}} \cdot \Delta \boldsymbol{P}^{\mathrm{se}}+\overline{\boldsymbol{\sigma}}^{\mathrm{e}}: \Delta \boldsymbol{\varepsilon}^{\mathrm{se}}>2 E_{0} P_{0} P .
$$

\section{Numerical examples}

The subsequent numerical examples are based on a three-dimensional finite element framework. A brief outline of the coupled formulation is summarized in Appendix A. We will assume that each crystallite is represented by one (eight-node brick) finite element and, moreover, consider the polarization vector and spontaneous strain tensor to remain uniform within one element. Conceptually speaking, $\boldsymbol{P}^{\mathrm{s}}$ and $\boldsymbol{\varepsilon}^{\mathrm{s}}$ are attached to individual elements rather than to integration points as commonly applied for internal variable formulations in computational inelasticity. The orientation initialization of the underlying unit-cells is perform via random generation as highlighted in Section 2.3 and the onset of domain switching follows the criterion developed in Eqs. (11), (16), (19). A staggered iteration scheme is applied for the subsequent finite element examples: (i) for given boundary/loading conditions, $\phi$ and $\boldsymbol{u}$ are computed at fixed $\boldsymbol{P}^{\mathrm{s}}, \boldsymbol{\varepsilon}^{\mathrm{s}}$ and $\boldsymbol{d}$, (ii) $\boldsymbol{P}^{\mathrm{s}}, \boldsymbol{\varepsilon}^{\mathrm{s}}$ and $\boldsymbol{d}$ are allowed to switch element-wise for given $\phi$ and $\boldsymbol{u}$, (iii) for identical boundary/loading conditions, $\phi$ and $\boldsymbol{u}$ are recomputed based on the updated values for $\boldsymbol{P}^{\mathrm{s}}, \boldsymbol{\varepsilon}^{\mathrm{s}}$ and $\boldsymbol{d}$; a detailed flowchart is summarized in Table 1 . As previously mentioned, the chosen polarization direction within the switched domain refers to that orientation among five kinematically admissible which (for given $\phi$ and $\boldsymbol{u}$ ) results in the largest energy reduction. Furthermore, all finite elements are allowed to switch at the same time. For computational efficiency (among other reasons), this iterative scheme is solely repeated once even though the finally computed state is not necessarily in electromechanical equilibrium in the sense that further switching might occur at the particular loading level. Nevertheless, the developed algorithm turns out to perform very robust and to render results which are in reasonably good agreement with experimental measurements.

In view of reproducing common experimental setups, a block-like specimen under electrical loading conditions is considered in the following. Essential boundary conditions are introduced by means of prescribing the electric potential at the top, $\phi_{\mathrm{top}}^{\mathrm{p}}$, and bottom surface, $\phi_{\mathrm{bot}}^{\mathrm{p}}$; see Fig. 5 for a graphical illustration. The electric potential at the bottom surface $\phi_{\text {bot }}^{\mathrm{p}}$ is thereby throughout zero while the loading itself is performed by incrementally increasing and decreasing $\phi_{\text {top }}^{\mathrm{p}}$ in a cyclic but quasi-static manner. Charge and traction free conditions are applied to the remaining surfaces the displacements at the bottom surface $\boldsymbol{u}_{\text {bot }}^{\mathrm{p}}$, however, being clamped. For illustration purpose of commonly studied hysteresis and butterfly curves and in order to compare the later on obtained numerical results with experimental data, volume averaged quantities of interest are projected onto the longitudinal electrical loading direction $\boldsymbol{e}_{3}$, namely

$$
\mathrm{D}=\boldsymbol{e}_{3} \cdot \frac{1}{\mathcal{V}} \int_{\mathcal{V}} \boldsymbol{D} \mathrm{d} v, \quad \mathrm{E}=\boldsymbol{e}_{3} \cdot \frac{1}{\mathcal{V}} \int_{\mathcal{V}} \boldsymbol{E} \mathrm{d} v, \quad \mathrm{e}=\boldsymbol{e}_{3} \cdot \frac{1}{\mathcal{V}} \int_{\mathcal{V}} \boldsymbol{\varepsilon} \mathrm{d} v \cdot \boldsymbol{e}_{3} .
$$


Table 1

Flowchart of the finite element algorithm: staggered iteration technique applied to piezoelectric switching

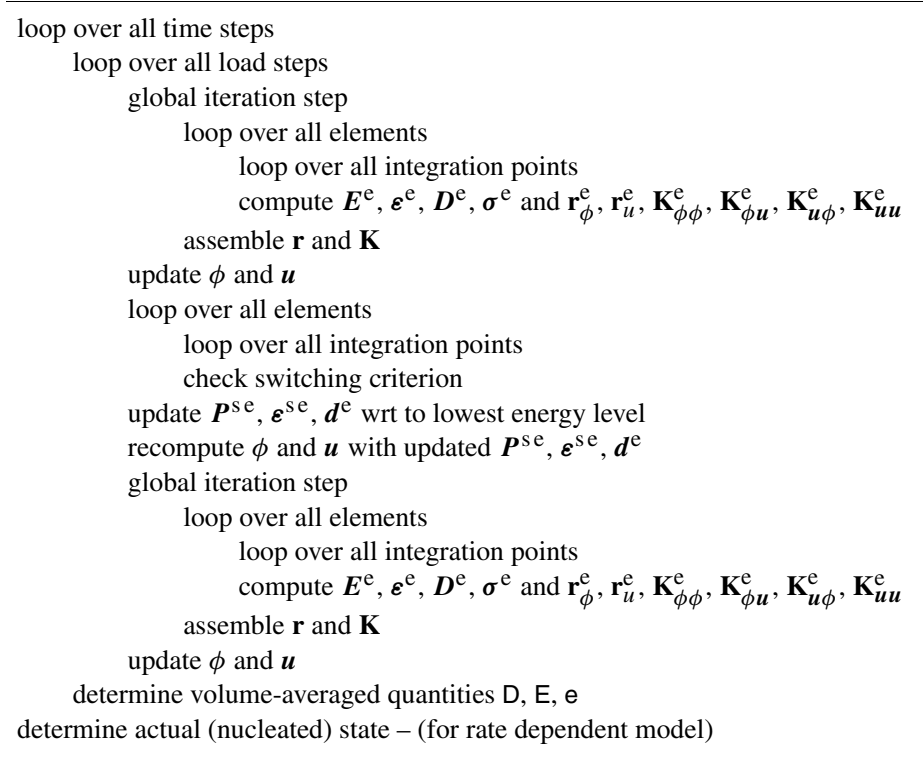

The subsequent finite element calculations refer to a block-like specimen arranged in a $9 \times 9 \times 9$ cube and its discretization has been performed with $9 \times 9 \times 9$ linear eight node bricks (Q1Q1) with the edges of the mesh being aligned with the global Cartesian frame $\left\{\boldsymbol{e}_{1,2,3}\right\}$, see Fig. 5. Standard material parameters for PIC 151 have been adopted from the literature: $\epsilon=0.0666[\mu \mathrm{F} / \mathrm{m}], d_{33}=1.52 \times 10^{-9}[\mathrm{~m} / \mathrm{V}], d_{31}=-0.57 \times 10^{-9}[\mathrm{~m} / \mathrm{V}], d_{15}=1.856 \times 10^{-9}[\mathrm{~m} / \mathrm{V}]$, Young's modulus $E=\mu[3 \lambda+2 \mu] /[\lambda+\mu]=30.3[\mathrm{GPa}]$, Poisson ration $v=\lambda /[2 \lambda+2 \mu]=0.3, \varepsilon^{\mathrm{s}}=2.7 \times 10^{-3}$, $P^{\mathrm{s}}=P_{0}=0.1938\left[\mathrm{C} / \mathrm{m}^{2}\right], E_{0}=0.4[\mathrm{MV} / \mathrm{m}]$, and, moreover, $C=0.003[\mathrm{MV} / \mathrm{m}]$ as well as $\left|\Delta \phi_{\text {top }}\right|=1.0[\mathrm{KV}]$. Concerning the boundary conditions, three different loading frequencies and amplitudes are chosen, i.e. $f_{\phi}=0.01$, $f_{\phi}=0.10, f_{\phi}=1.00[\mathrm{~Hz}]$ and $\widehat{\mathrm{E}}=2.0, \widehat{\mathrm{E}}=1.5, \widehat{\mathrm{E}}=1.0[\mathrm{MV} / \mathrm{m}]$.

Numerical examples studied in the progression of this work exclusively deal with rate-dependent response even though rate-independent modeling is naturally included within the proposed formulation; compare Sections 3.1 and 3.2. For detailed studies of rate-independent behavior we refer the reader to Arockiarajan et al. (2005b) where compressive loading stresses are additionally addressed. Application of axial and lateral mechanical loading (besides longitudinal electrical loading) to the rate-dependent formulation for piezoelectric materials developed in this contribution constitutes future research.

\subsection{Simulation of rate-dependent switching without grain boundary effects}

Incorporation of grain boundary effects is, for comparison reasons, neglected in this section, i.e. we throughout choose $P=1$ or rather $k=0$.

Figs. 6-8 show hysteresis and butterfly curves for various loading amplitudes and frequencies. Apparently, the hysteresis curves possess sharp corners near the macroscopic coercive electric field which is commonly not observed in experiments. It is, however, experimentally verified that the macroscopic coercive electric field depends on the loading frequency which is clearly captured by the numerical results displayed in Figs. 6-8 since the simulated macroscopic coercive electric field increases with increasing loading frequencies irrespective of the loading amplitudes. This increase is even observed for an electric field loading amplitude of $\widehat{E}=1.0[\mathrm{MV} / \mathrm{m}]$ which is in the range of the macroscopic coercive electric field itself, see Fig. 8. Another important observation is that the electric displacements and longitudinal strains decrease for higher loading frequencies - even if the amplitude of the applied electric field decreases. Furthermore, Figs. 6 and 7 additionally underpin that the electric displacements and total strain evolution is not entirely saturated for a loading frequency of $1.00[\mathrm{~Hz}]$ compared with the almost quasi-static loading cases at 0.10 or $0.01[\mathrm{~Hz}]$. Practically speaking, the electric displacements and total strains rather increase at points where the 

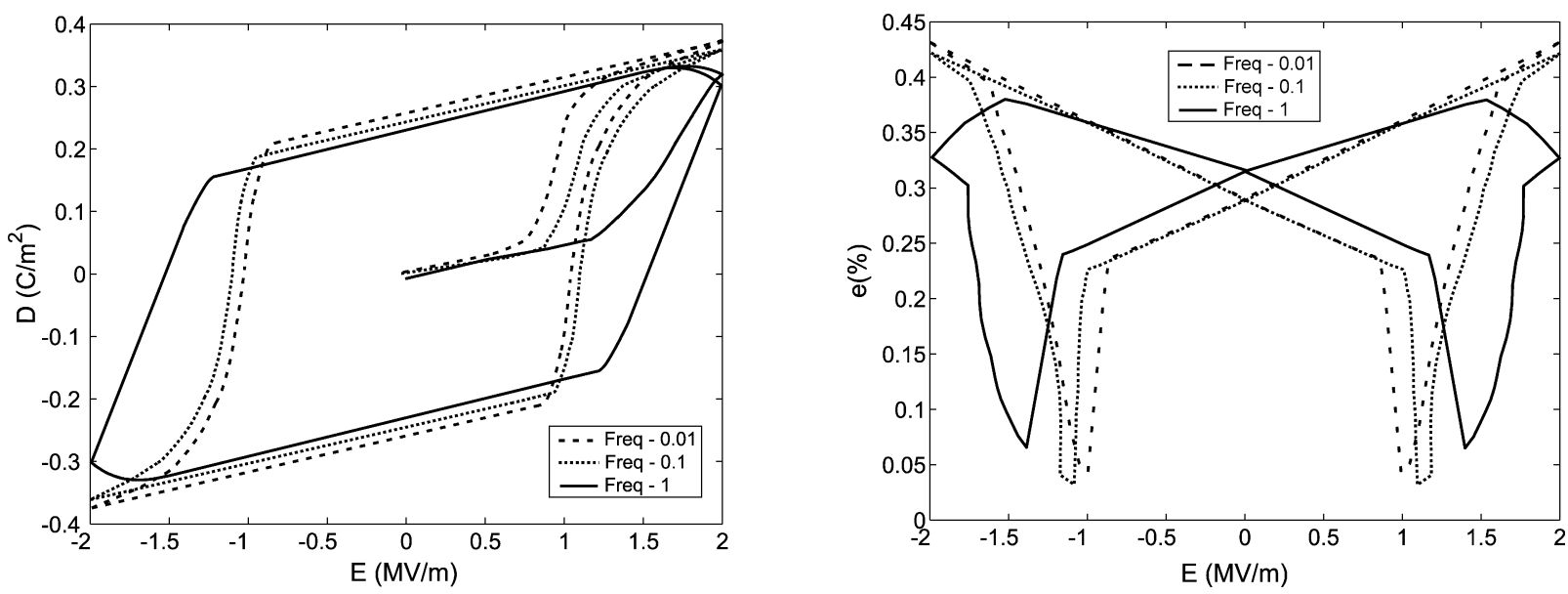

Fig. 6. Hysteresis and butterfly curves without probability function $(k=0) ; \widehat{E}=2.0[\mathrm{MV} / \mathrm{m}]$.
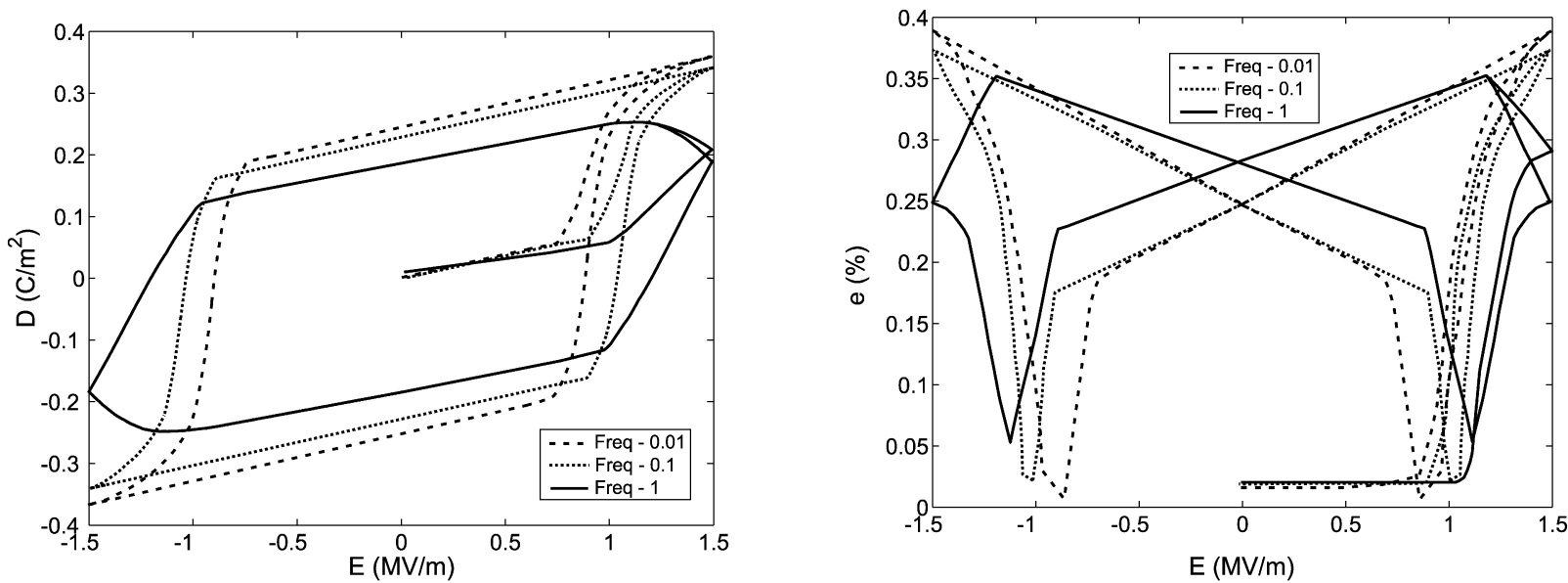

Fig. 7. Hysteresis and butterfly curves without probability function $(k=0) ; \widehat{\mathrm{E}}=1.5[\mathrm{MV} / \mathrm{m}]$.
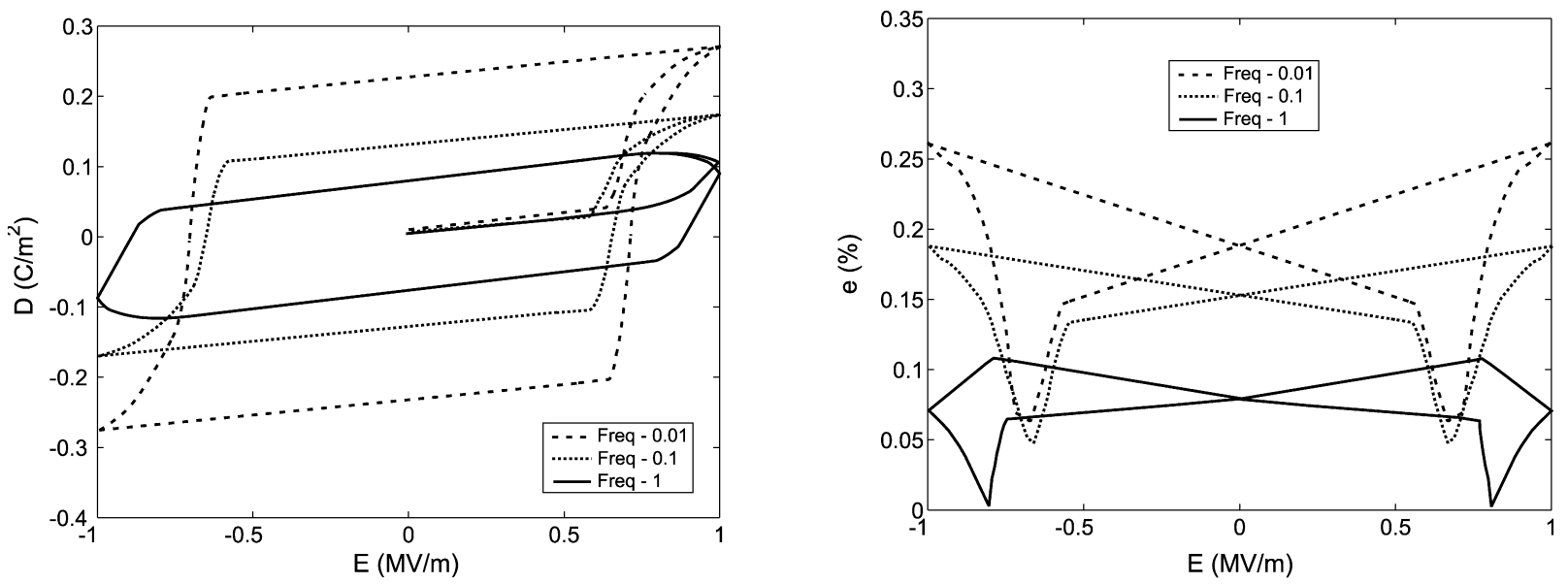

Fig. 8. Hysteresis and butterfly curves without probability function $(k=0) ; \widehat{\mathrm{E}}=1.0[\mathrm{MV} / \mathrm{m}]$. 

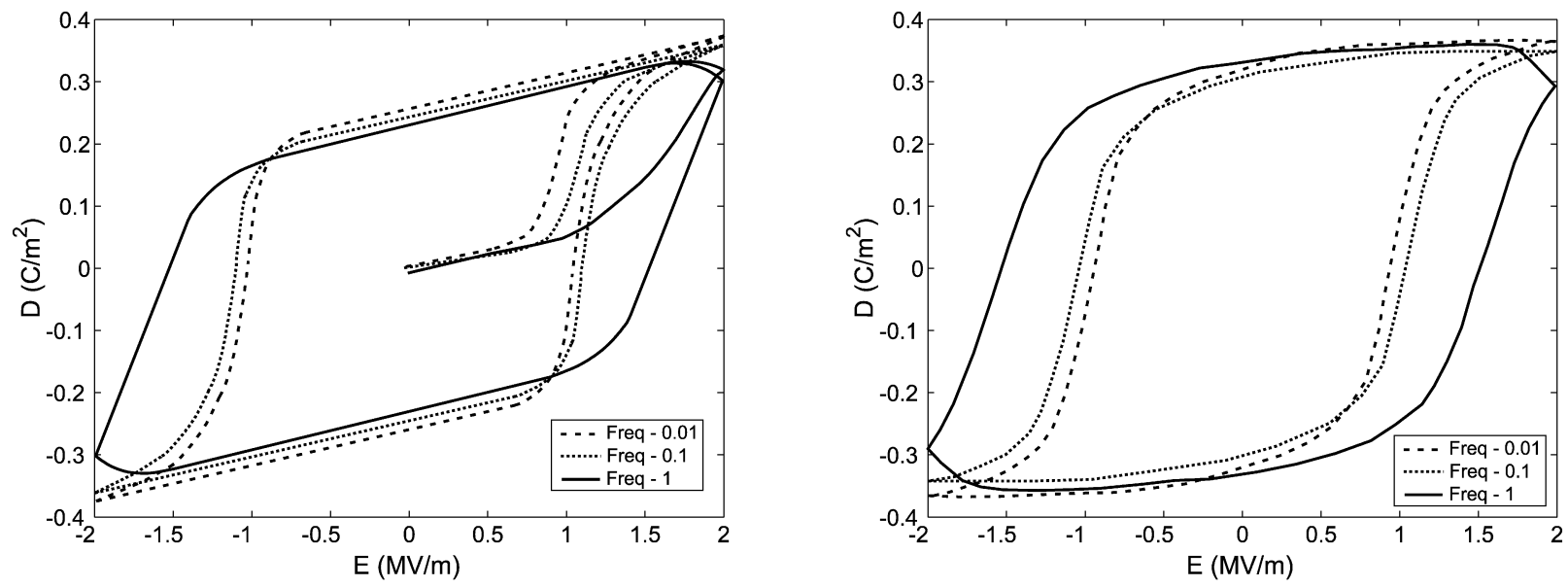

Fig. 9. Hysteresis curve with fifth order probability function $(k=5)$, (left) and experimental data - Zhou et al. (2001), (right); $\widehat{E}=2.0[M V / m]$.
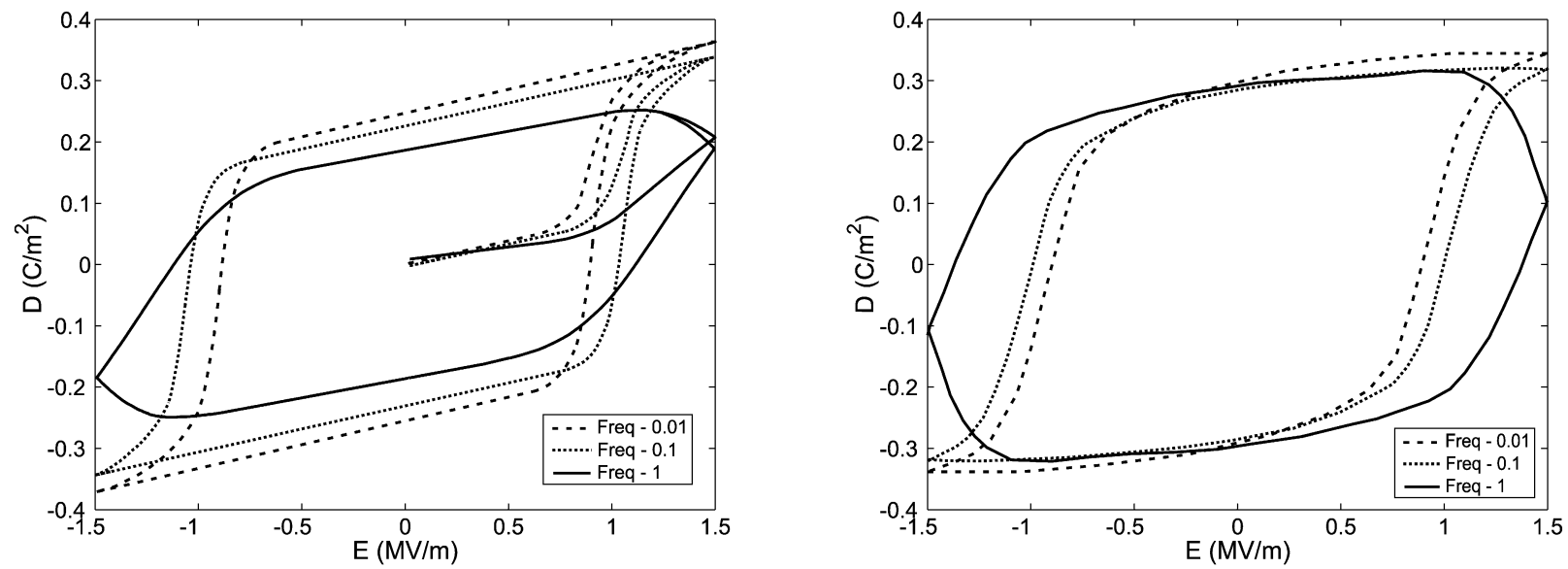

Fig. 10. Hysteresis curve with fifth order probability function $(k=5)$, (left) and experimental data - Zhou et al. (2001), (right); $\widehat{E}=1.5[\mathrm{MV} / \mathrm{m}]$.
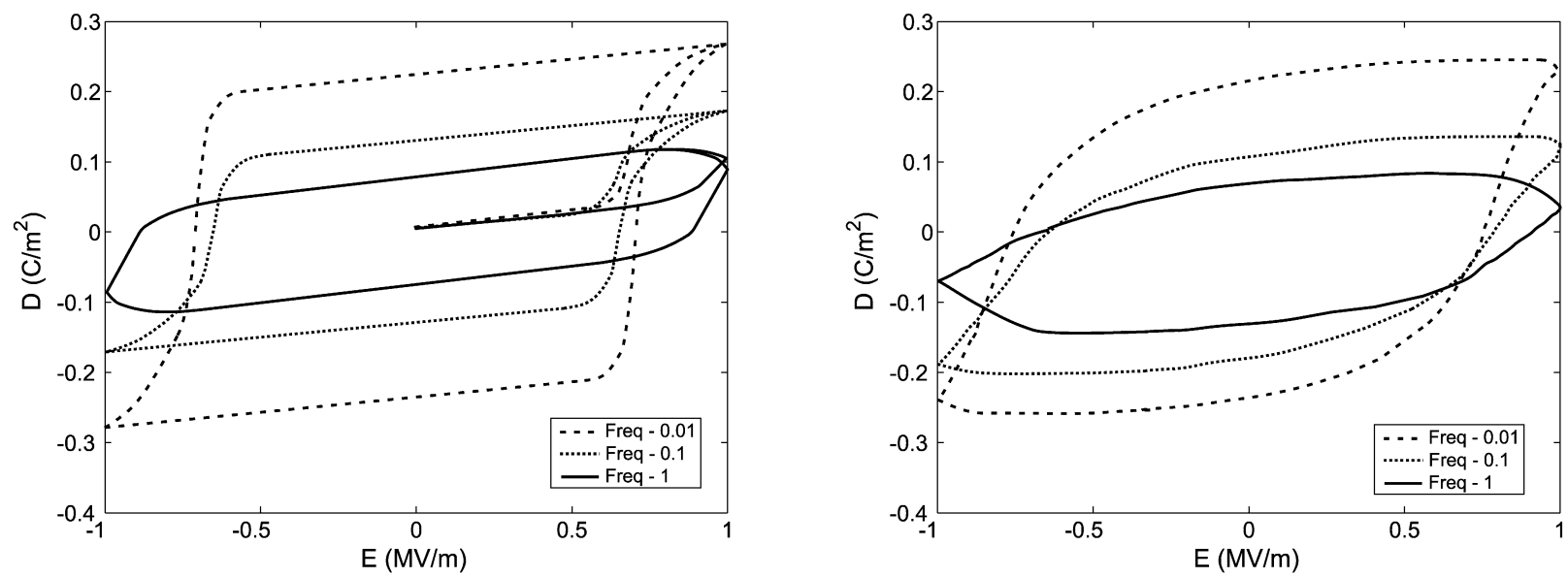

Fig. 11. Hysteresis curve with fifth order probability function $(k=5)$, (left) and experimental data - Zhou et al. (2001), (right); $\widehat{E}=1.0[\mathrm{MV} / \mathrm{m}]$. 

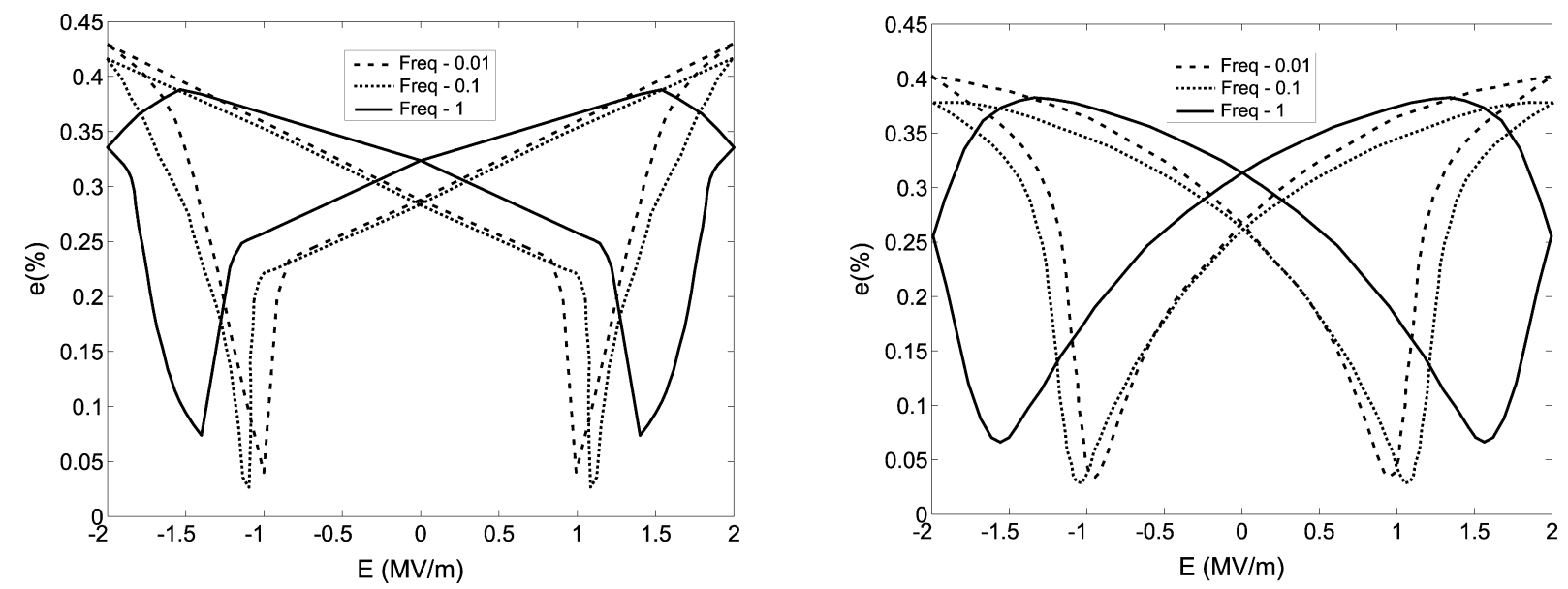

Fig. 12. Butterfly curve with fifth order probability function $(k=5)$, (left) and experimental data - Zhou et al. (2001), (right); $\widehat{E}=2.0[M V / m]$.
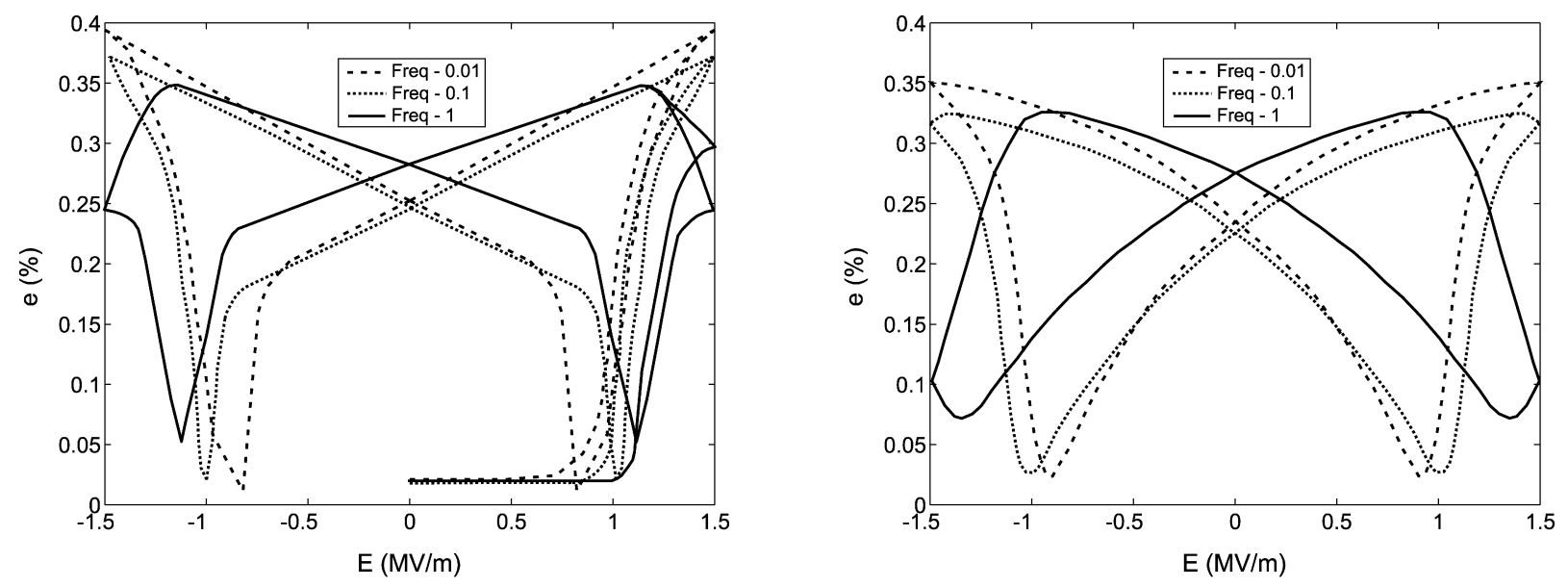

Fig. 13. Butterfly curve with fifth order probability function $(k=5)$, (left) and experimental data - Zhou et al. (2001), (right); $\widehat{\mathrm{E}}=1.5[\mathrm{MV} / \mathrm{m}]$.
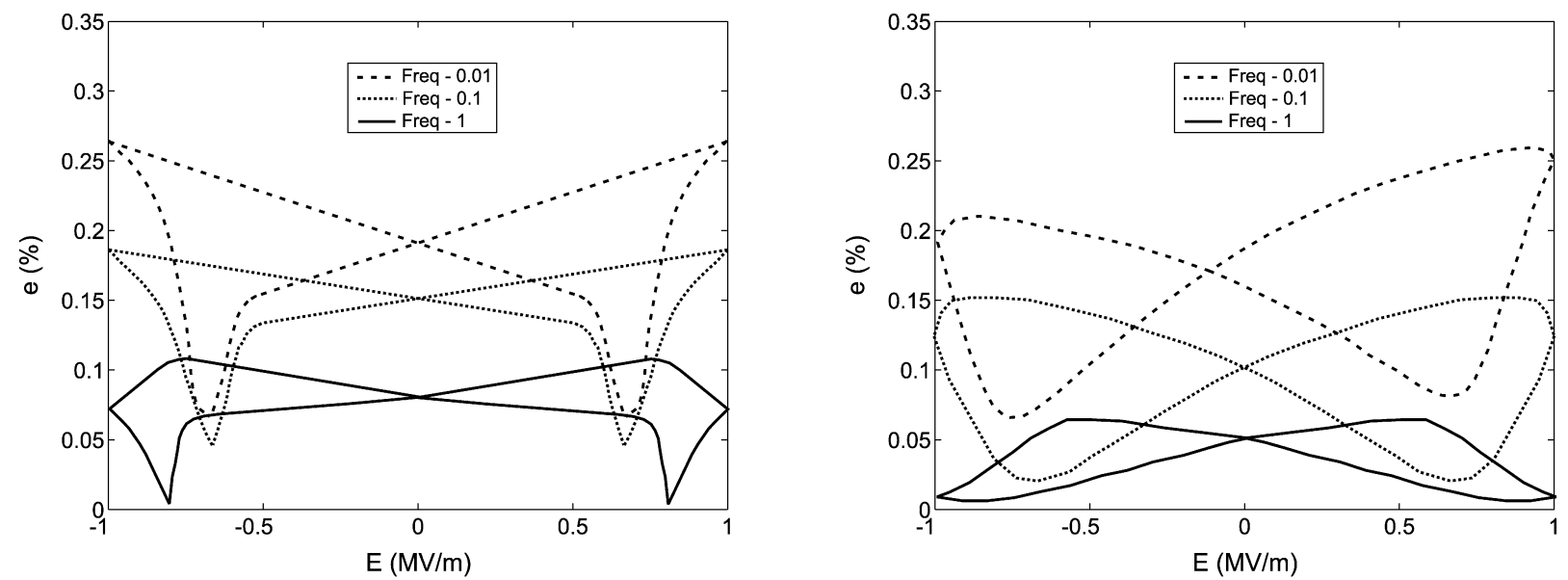

Fig. 14. Butterfly curve with fifth order probability function $(k=5)$, (left) and experimental data - Zhou et al. (2001), (right); $\widehat{\mathrm{E}}=1.0[\mathrm{MV} / \mathrm{m}]$. 
loading direction is reversed $(\widehat{E}=2.0,1.5,1.0[\mathrm{MV} / \mathrm{m}])$. In addition, Figs. 6 and 7 monitor that the remanent polarization (electrical displacement at zero electric field) and remanent total strains (longitudinal strain at zero electric field) values are considerably un-affected at loading frequencies $f_{\phi}$ of 0.01 and $0.10[\mathrm{~Hz}]$ and electric field amplitudes $\widehat{E}$ of 2.0 and $1.5[\mathrm{MV} / \mathrm{m}]$. The main reason for this effect stems from the fact that possible domain switching has widely been completed within one representative time step. From Fig. 8, however, it is clearly observed that the remanent polarization and remanent strains decrease with increasing loading frequencies.

\subsection{Simulation of rate-dependent switching with grain boundary effects}

Within the subsequent example, intergranular effects are phenomenologically incorporated via the proposed switching probability function. The additional material parameter or rather the polynomial degree of the probability function has thereby been chosen as $k=5$ which renders the simulations to nicely match experimental data.

By analogy with Figs. 6-8 in the previous section, Figs. 9(left)-14(left) show computed hysteresis and butterfly curves for various loading frequencies and amplitudes while Figs. 9(right)-14(right) monitor experimental data reported by Zhou et al. (2001). Since intergranular effects have been taken into account, the simulated curves are much smoother near the macroscopic coercive field compared to the results in Section 4.1. The curves displayed in Figs. 9(left)-14(left) reveal similar observations as those in Figs. 6-8, for instance: the coercive electric field increases with increasing loading frequencies; the remanent polarization and remanent total strains increase at lower loading amplitudes; etc. As a general rule, electric displacements and total strains increase at higher loading frequencies until the coercive field is reached. Apparently, the computations including the probabilistic approach better match experimental results and are in good agreement with the results reported by Zhou et al. (2001).

\section{Summary}

The main goal of this contribution was the development of a robust coupled three-dimensional finite element formulation capturing rate-dependent switching effects of piezoelectric materials. As a key feature of the proposed framework, a micromechanically motivated energy-based switching criterion has been applied which accounts for coupled electromechanical loading. Besides a phenomenological approach for the incorporation of intergranular effects, a particular volume fraction concept or rather linear kinetics theory enabled the incorporation of rate-dependent switching effects. Based on these elaborations and an initially random polarization orientation to model the un-poled state, finite element examples have been studied. We thereby assumed individual grains to be represented by single finite elements and made use of a staggered iteration scheme. Representative macroscopic hysteresis and butterfly curves have been computed for various loading frequencies and amplitudes by additionally adopting standard volume averaging techniques. As a result, the performed simulations could be compared with measured data and it turned out that the computations based on the developed formulation nicely match experiments. Future research will place particular emphasis on the modeling of rate-dependent switching under coupled electromechanical loading conditions.

\section{Acknowledgements}

The authors thank the 'Deutsche Forschungsgemeinschaft (DFG)' for financial support of this work within the 'Graduiertenkolleg 814, Ingenieurmaterialien auf verschiedenen Skalen: Experiment, Modellierung und Simulation'.

\section{Appendix A. Finite element setting}

For convenience of the reader, we briefly review the applied electromechanically coupled finite element formulation in the following whereby $\phi$ and $\boldsymbol{u}$ constitute the essential degrees of freedom. Adopting standard concepts from computational inelasticity, the local orthonormal frame $\left\{\boldsymbol{m}_{1,2,3}\right\}$ is thereby stored at the integration point or rather element level, from which both the polarization vectors as well as the spontaneous strain tensor can be computed. The 
finite element formulation itself is directly based on the principle of virtual work so that the correlated weak forms read

$$
\begin{aligned}
G_{\phi} & =-\int_{\mathcal{B}} \delta \boldsymbol{E} \cdot \boldsymbol{D} \mathrm{d} v-\int_{\partial \mathcal{B}_{D}} \delta \phi q \mathrm{~d} a=G_{\phi}^{\mathrm{int}}-G_{\phi}^{\mathrm{ext}}=0, \\
G_{\boldsymbol{u}} & =\int_{\mathcal{B}} \delta \boldsymbol{\varepsilon}: \boldsymbol{\sigma} \mathrm{d} v-\int_{\partial \mathcal{B}_{\sigma}} \delta \boldsymbol{u} \cdot \boldsymbol{t} \mathrm{d} a=G_{\boldsymbol{u}}^{\mathrm{int}}-G_{\boldsymbol{u}}^{\mathrm{ext}}=0
\end{aligned}
$$

wherein $\boldsymbol{b}$ and $\rho_{f}$ have been assumed to vanish, compare Eq. (1). Adopting standard finite element techniques, one obtains linearized (incremental) representations of Eqs. (A.1) at the element level and for conservative loading as

$$
\begin{aligned}
& \Delta G_{\phi}^{\mathrm{e}}=-\int_{\mathcal{B}^{\mathrm{e}}} \delta \boldsymbol{E} \cdot\left[\boldsymbol{d}:\left[\Delta \boldsymbol{\varepsilon}-\Delta \boldsymbol{\varepsilon}^{\mathrm{s}}\right]+\Delta \boldsymbol{d}:\left[\boldsymbol{\varepsilon}-\boldsymbol{\varepsilon}^{\mathrm{s}}\right]+\boldsymbol{\epsilon} \cdot \Delta \boldsymbol{E}+\Delta \boldsymbol{P}^{\mathrm{s}}\right] \mathrm{d} v, \\
& \Delta G_{\boldsymbol{u}}^{\mathrm{e}}=\int_{\mathcal{B}^{\mathrm{e}}} \delta \boldsymbol{\varepsilon}:\left[\mathbf{C}:\left[\Delta \boldsymbol{\varepsilon}-\Delta \boldsymbol{\varepsilon}^{\mathrm{s}}\right]-\Delta \boldsymbol{E} \cdot \boldsymbol{d}-\boldsymbol{E} \cdot \Delta \boldsymbol{d}\right] \mathrm{d} v .
\end{aligned}
$$

Based on standard (iso-parametric) interpolation, the approximated electric potential $\phi$ and $\delta \phi, \Delta \phi$ as well as the displacement field $\boldsymbol{u}$ and $\delta \boldsymbol{u}, \Delta \boldsymbol{u}$ result in

$$
\begin{aligned}
& \phi^{\mathrm{h}}=\sum_{i=1}^{n_{\text {nod }}} N_{i} \phi_{i}, \quad \delta \phi^{\mathrm{h}}=\sum_{i=1}^{n_{\text {nod }}} N_{i} \delta \phi_{i}, \quad \Delta \phi^{\mathrm{h}}=\sum_{i=1}^{n_{\text {nod }}} N_{i} \Delta \phi_{i}, \\
& \boldsymbol{u}^{\mathrm{h}}=\sum_{i=1}^{n_{\text {nod }}} N_{i} \boldsymbol{u}_{i}, \quad \delta \boldsymbol{u}^{\mathrm{h}}=\sum_{i=1}^{n_{\text {nod }}} N_{i} \delta \boldsymbol{u}_{i}, \quad \Delta \boldsymbol{u}^{\mathrm{h}}=\sum_{i=1}^{n_{\text {nod }}} N_{i} \Delta \boldsymbol{u}_{i}
\end{aligned}
$$

with $\boldsymbol{x}^{\mathrm{h}}=\sum_{i=1}^{n_{\text {nod }}} N_{i} \boldsymbol{x}_{i}$ being obvious and, moreover, $n_{\text {nod }}$ and $N_{i}$ denoting the number of nodes per element as well as the shape functions, respectively. Application of the kinematic relations mentioned in Section 2.2 together with Eq. (A.3) consequently enables the following approximations

$$
\begin{array}{lll}
\boldsymbol{E}^{\mathrm{h}}=-\sum_{i=1}^{n_{\text {nod }}} \phi_{i} \nabla N_{i}, & \delta \boldsymbol{E}^{\mathrm{h}}=-\sum_{i=1}^{n_{\text {nod }}} \delta, \phi_{i} \nabla N_{i}, & \Delta \boldsymbol{E}^{\mathrm{h}}=-\sum_{i=1}^{n_{\text {nod }}} \Delta \phi_{i} \nabla N_{i}, \\
\boldsymbol{\varepsilon}^{\mathrm{h}}=\sum_{i=1}^{n_{\text {nod }}}\left[\boldsymbol{u}_{i} \otimes \nabla N_{i}\right]^{\mathrm{sym}}, & \delta \boldsymbol{\varepsilon}^{\mathrm{h}}=\sum_{i=1}^{n_{\text {nod }}}\left[\delta \boldsymbol{u}_{i} \otimes \nabla N_{i}\right]^{\mathrm{sym}}, & \Delta \boldsymbol{\varepsilon}^{\mathrm{h}}=\sum_{i=1}^{n_{\text {nod }}}\left[\Delta \boldsymbol{u}_{i} \otimes \nabla N_{i}\right]^{\mathrm{sym}}
\end{array}
$$

so that the discrete format for $G_{\phi}$ and $G_{\boldsymbol{u}}$ allows representations as

$$
\begin{aligned}
G_{\phi}^{\mathrm{e}} & =\int_{\mathcal{B}^{\mathrm{e}}} \sum_{i=1}^{n_{\text {nod }}} \delta \phi_{i}\left[\nabla N_{i} \cdot \boldsymbol{D}\right] \mathrm{d} v-\int_{\partial \mathcal{B}_{D}^{\mathrm{e}}} \sum_{i=1}^{n_{\text {nod }}} \delta \phi_{i}\left[N_{i} q\right] \mathrm{d} a=0, \\
G_{\boldsymbol{u}}^{\mathrm{e}} & =\int_{\mathcal{B}^{\mathrm{e}}} \sum_{i=1}^{n_{\text {nod }}} \delta \boldsymbol{u}_{i} \cdot\left[\nabla N_{i} \cdot \boldsymbol{\sigma}\right] \mathrm{d} v-\int_{\partial \mathcal{B}_{\sigma}^{\mathrm{e}}} \sum_{i=1}^{n_{\text {nod }}} \delta \boldsymbol{u}_{i} \cdot\left[N_{i} \boldsymbol{t}\right] \mathrm{d} a=0
\end{aligned}
$$

and, by analogy with Eq. (A.2), we finally obtain

$$
\begin{aligned}
& \Delta G_{\phi}^{\mathrm{e}}=-\int_{\mathcal{B}^{\mathrm{e}}} \sum_{i, j=1}^{n_{\text {nod }}} \delta \phi_{i}\left[\nabla N_{i} \cdot \boldsymbol{\epsilon} \cdot \nabla N_{j}\right] \Delta \phi_{j}+\delta \phi_{i}\left[\nabla N_{i} \cdot \boldsymbol{d} \cdot \nabla N_{j}\right] \cdot \Delta \boldsymbol{u}_{j} \mathrm{~d} v, \\
& \Delta G_{\boldsymbol{u}}^{\mathrm{e}}=-\int_{\mathcal{B}^{\mathrm{e}}} \sum_{i, j=1}^{n_{\text {nod }}} \delta \boldsymbol{u}_{i} \cdot\left[\nabla N_{i} \cdot \boldsymbol{d} \cdot \nabla N_{j}\right] \Delta \phi_{j}-\delta \boldsymbol{u}_{i} \cdot\left[\nabla N_{i} \cdot \mathbf{C} \cdot \nabla N_{j}\right] \cdot \Delta \boldsymbol{u}_{j} \mathrm{~d} v .
\end{aligned}
$$

Any contributions stemming from $\Delta \boldsymbol{P}^{\mathrm{s}}, \Delta \boldsymbol{\varepsilon}^{\mathrm{s}}$ or $\Delta \boldsymbol{d}$ are neglected in Eq. (A.6) but explicitly captured within the staggered iteration scheme summarized in Table 1. In view of a correlated finite element implementation, the terms in 
brackets in Eqs. (A.5), (A.6) render, after assembly with respect to the global finite element setting, the residual vectors $\left(\mathbf{r}_{\phi}\right.$ and $\left.\mathbf{r}_{\boldsymbol{u}}\right)$ as well as incremental tangent stiffness matrix contributions $\left(\mathbf{K}_{\phi \phi}, \mathbf{K}_{\phi \boldsymbol{u}}, \mathbf{K}_{\boldsymbol{u} \phi}\right.$ and $\left.\mathbf{K}_{\boldsymbol{u} \boldsymbol{u}}\right)$, respectively.

\section{References}

Abeyaratne, R., Kim, S.J., Knowles, J.K., 1994. One dimensional model for shape memory alloys. Int. J. Solids Struct. 31, $2229-2249$.

Allik, H., Hughes, T.J.R., 1970. Finite element method for piezoelectric vibration. Int. J. Numer. Methods Engrg. 2, $151-157$.

Arlt, G., 1996a. Switching and dielectric nonlinearity of ferroelectric ceramics. Ferroelectrics 189, 91-101.

Arlt, G., 1996b. A physical model for hysteresis curves of ferroelectric ceramics. Ferroelectrics 189, 103-119.

Arockiarajan, A., Menzel, A., Delibas, B., Seemann, W., 2005a. Studies on rate-dependent switching effects of piezoelectric materials using a finite element model. Comput. Mater. Sci., available online.

Arockiarajan, A., Delibas, B., Menzel, A., Seemann, W., 2005b. Micromechanical modeling of switching effects in piezoelectric materials - a robust coupled finite element approach, submitted for publication.

Cao, H., Evans, A.G., 1993. Nonlinear deformation of ferroelectric ceramics. J. Am. Ceram. Soc. 76, 890-896.

Chen, X., Fang, D.N., Hwang, K.C., 1997. Micromechanics simulation of ferroelectric polarization switching. Acta Mater. 45, 3181-3189.

Chen, W., Lynch, C.S., 1998. A micro-electro-mechanical model for polarization switching of ferroelectric materials. Acta Mater. 46, 5303-5311.

Delibas, B., Arockiarajan, A., Seemann, W., 2006. Rate dependent properties of perovskite type tetragonal piezoelectric materials using micromechanical model. Int. J. Solids Struct. 43, 697-712.

Fotinich, Y., Carman, G.P., 2000. Stresses in piezoceramics undergoing polarization switchings. J. Appl. Phys. 88, 6715-6725.

Gaudenzi, P., Bathe, K.J., 1995. An iterative finite element procedure for the analysis of piezoelectric continua. J. Intel. Syst. Str. 6, $266-273$.

Goldstein, H., Poole, C., Safko, J., 2002. Classical Mechanics, third ed. Addison-Wesley, New York.

Huber, J.E., Fleck, N.A., 2004. Ferroelectric switching: a micromechanics model versus measured behaviour. Eur. J. Mech. A Solids 23, $203-217$.

Hwang, S.C., Lynch, C.S., McMeeking, R.M., 1995. Ferroelectric/ferroelastic interactions and a polarization switching model. Acta Metall. Mater. 43, 2073-2084.

Hwang, S.C., McMeeking, R.M., 1998a. The prediction of switching in polycrystalline ferroelectric ceramics. Ferroelectrics 207, 465-495.

Hwang, S.C., McMeeking, R.M., 1998b. A finite element model of ferroelectric polycrystals. Ferroelectrics 211, 177-194.

Janta, J., 1971. The influence of the shape of domains on the ferroelectric hysteresis loop. Ferroelectrics 2, 299-302.

Kamlah, M., Böhle, U., 2001. Finite element analysis of piezoceramic components taking into account ferroelectric hysteresis behaviour. Int. J. Solids Struct. 38, 605-633.

Kamlah, M., Tsakmakis, C., 1999. Phenomenological modeling of non-linear electromechanical coupling in ferroelectrics. Int. J. Solids Struct. 36, $666-695$.

Landis, C.M., 2002. Fully coupled, multi-axial, symmetric constitutive laws for polycrystalline ferroelectric ceramics. J. Mech. Phys. Solids 50, $127-152$

Li, W.F., Weng, G.J., 2001. Micromechanics simulation of spontaneous polarization in ferroelectric crystals. J. Appl. Phys. 90, $2484-2491$.

Lu, W., Fang, D.-N., Li, C.Q., Hwang, K.C., 1999. Nonlinear electric-mechanical behaviour and micromechanics modeling of ferroelectric domain evolution. Acta Mater. 47, 2913-2926.

Lynch, C.S., 1996. The effect of uniaxial stress on the electro-mechanical response of 8/65/35 PLZT. Acta Mater. 44, 4137-4148.

Lynch, C.S., McMeeking, R.M., 1994. Finite strain ferroelectric constitutive laws. Ferroelectrics 160, 177-184.

McMeeking, R.M., Hwang, S.C., 1997. On the potential energy of a piezoelectric inclusion and the criterion for ferroelectric switching. Ferroelectrics 200, 151-173.

Merz, W.J., 1954. Domain formation and domain wall motions in ferroelectric $\mathrm{BaTiO}_{3}$ single crystals. Phys. Rev. 95 (3), $690-698$.

Schröder, J., Gross, D., 2004. Invariant formulation of the electromechanical enthalpy function of transversely isotropic piezoelectric materials Arch. Appl. Mech. 73, 533-552.

Schröder, J., Romanowski, H., 2005. A thermodynamically consistent mesoscopic model for transversely isotropic ferroelectric ceramics in a coordinate-invariant setting. Arch. Appl. Mech. 74, 863-877.

Silvester, P.P., Ferrari, R.L., 1996. Finite Elements for Electrical Engineers. Cambridge University Press.

Smith, R.C., Ounaies, Z., 2000. Domain wall model for hysteresis in piezoelectric materials. J. Intel. Mat. Syst. Str. 11, 62-79.

Zhou, D., Kamlah, M., Munz, D., 2001. Rate dependence of soft PZT ceramics under electric field loading. Proc. SPIE 4333 (11), 64-70. 